\title{
Homéoprotéines et plasticité cellulaire / Homeoproteins and cell plasticity
}

Centre interdisciplinaire de recherche en biologie (CIRB)

\section{Sophie Vriz et Alain Joliot}

\section{(2) OpenEdition}

\section{Journals}

Édition électronique

URL : https://journals.openedition.org/annuaire-cdf/16188

DOI : 10.4000/annuaire-cdf. 16188

ISBN : 978-2-7226-0572-5

ISSN : 2109-9227

\section{Éditeur}

Collège de France

\section{Édition imprimée}

Date de publication : 30 décembre 2020

Pagination : $672-673$

ISBN : 978-2-7226-0516-9

ISSN : 0069-5580

\section{Référence électronique}

Sophie Vriz et Alain Joliot, " Homéoprotéines et plasticité cellulaire / Homeoproteins and cell plasticity », L'annuaire du Collège de France [En ligne], 118 | 2020, mis en ligne le 01 avril 2021, consulté le 22 août 2022. URL : http://journals.openedition.org/annuaire-cdf/16188; DOI : https://doi.org/10.4000/ annuaire-cdf. 16188 
Chaigne A., Terret M.-É. et Verlhac M.-H., « Asymmetries and symmetries in the mouse oocyte and zygote », Results and Problems in Cell Differentiation, vol. 61, 2017, p. 285-299, DOI : 10.1007/978-3-319-53150-2_13.

VERLHAC M.-H., "An actin shell delays oocyte chromosome capture by microtubules », Journal of Cell Biology, vol. 217, no 8, 2018, p. 2601-2603, DOI : 10.1083/jcb.201807016.

\title{
HOMÉOPROTÉINES ET PLASTICITÉ CELLULAIRE / HOMEOPROTEINS AND CELL PLASTICITY
}

\author{
Responsables : Sophie VRIZ et Alain JOLIOT
}

\section{RECHERCHE - RÉGULATION DE LA SÉCRÉTION CONVENTIONNELLE ET NON CONVENTIONNELLE DES PROTÉINES}

Page web : https://www.college-de-france.fr/site/en-cirb/joliot-vriz.htm.

Le peroxyde d'hydrogène $\left(\mathrm{H}_{2} \mathrm{O}_{2}\right)$ est aujourd'hui considéré comme un élément clé de la signalisation cellulaire. Sa production et sa dégradation sont finement régulées par chaque cellule. Il contribue à la régulation de processus physiologiques notamment en contrôlant le degré d'oxydation des cystéines au sein des protéines. L'élévation transitoire de $\mathrm{H}_{2} \mathrm{O}_{2}$ est requise pour induire des programmes de régénération chez les vertébrés, améliorer la plasticité cellulaire et favoriser la cicatrisation. Suite à la démonstration du rôle fondamental d' $\mathrm{H}_{2} \mathrm{O}_{2}$ dans les processus de régénération chez le poisson zèbre, nous avons démontré cette année la conservation de tels mécanismes chez les mammifères (Labit et al., 2018). Nous avons développé différentes stratégies pour manipuler les niveaux d' $\mathrm{H}_{2} \mathrm{O}_{2}$ dans le but de mimer la dynamique extrême des niveaux de $\mathrm{H}_{2} \mathrm{O}_{2}$ observée au cours du développement embryonnaire et de la régénération chez l'adulte. De plus, le ciblage de compartiments subcellulaires spécifiques nous permet d'analyser le rôle d' $\mathrm{H}_{2} \mathrm{O}_{2}$ dans le trafic des protéines, notamment des processus de sécrétion des protéines, conventionnelle ou non conventionnelle, tant ex vivo qu'in vivo.

La protéine Sonic Hedgehog (Shh) est un morphogène indispensable à l'initiation de la régénération, notamment par le contrôle des niveaux redox chez l'adulte (Meda et al., 2018 pour revue). En retour, nos résultats préliminaires montraient un effet de la signalisation redox dans la régulation de la sécrétion de Shh (Meda et al., 2018 pour revue). Afin d'étudier plus en détail l'impact de la signalisation redox sur la sécrétion de Shh, nous avons, d'une part, adapté des outils permettant la synchronisation de la sécrétion de Shh et, d'autre part, effectué des mutagenèses ciblées d'acides aminés susceptibles d'être la cible de la signalisation redox.

Certaines voies de sécrétion non conventionnelles impliquent un transfert direct du substrat sécrété au travers de la membrane plasmique. C'est le cas des homéoprotéines, qui, de plus, présentent la particularité de traverser la membrane dans le sens opposé, permettant ainsi leur internalisation et donc, leur transfert entre cellules. Nous avions démontré le rôle du phospholipide PIP2, enrichi dans la face interne de la membrane plasmique, dans la sécrétion des homéoprotéines. Ce même 
lipide est également nécessaire à l'internalisation de ces dernières, suggérant ainsi une forte similitude entre les mécanismes de transfert au travers de la membrane dans des sens opposés. Nous avons récemment observé que l'internalisation des homéoprotéines est aussi modulée par les niveaux redox cellulaires. La membrane plasmique étant un site privilégié de production d' $\mathrm{H}_{2} \mathrm{O}_{2}$, nous testons l'hypothèse d'une régulation des taux de PIP2 par la signalisation redox.

Au cours de cette année, nous avons renforcé notre collaboration avec l'équipe de Ludovic Jullien (ENS, Paris), ce qui a conduit à la validation d'une nouvelle méthode de quantification de la sécrétion des protéines (Li et al., 2018).

\section{Publications}

Meda F., Joliot A. et VRIZ S., « Nerves and hydrogen peroxide: How old enemies become new friends ", Neural Regeneration Research, vol. 12, $\mathrm{n}^{\mathrm{o}}$ 4, 2017, p. 568-569, DOI : 10.4103/1673-5374.205088.

Feng Z., NAm S., Hamouri F., Aujard I., Ducos B., VRiz S., Volovitch M., Jullien L., LiN S., WeISS S. et BENSIMON D., "Optical control of tumor induction in the zebrafish ", Scientific Reports, vol. 7, nº 1, 2017, p. 9195, DOI : 10.1038/s41598-017-09697-x.

Zhang W., Hamouri F., Feng Z., Aujard I., Ducos B., Ye S., Weiss S., Volovitch M., VRIZ S., JULLIEN L. et BENSIMON D., «Control of protein activity and gene expression by cyclofen-OH uncaging », Chembiochem: A European Journal of Chemical Biology, vol. 19, $\mathrm{n}^{\mathrm{o}}$ 12, 2018, p. 1232-1238, DOI : 10.1002/cbic.201700630.

Meda F., Rampon C., Dupont E., Gauron C., Mourton A., Queguiner I., Thauvin M., Volovitch M., Joliot A. et VRIZ S., « Nerves, $\mathrm{H}_{2} \mathrm{O}_{2}$ and Shh: Three players in the game of regeneration », Seminars in Cell and Developmental Biology, vol. 80, 2017, p. 65-73, DOI : 10.1016/j.semcdb.2017.08.015.

VRIZ S., «Redox signalling in development and regeneration», Seminars in Cell \& Developmental Biology, vol. 80, 2018, p. 1-2, DOI : 10.1016/j.semcdb.2017.09.011.

Li C., Mourton A., Plamont M.-A., Rodrigues V., Aujard I., Volovitch M., Le SauX T., Perez F., VRiz S., Jullien L., Joliot A. et Gautier A., « Fluorogenic probing of membrane protein trafficking », Bioconjugate Chemistry, vol. 29, n 6 , 2018, p. 1823-1828, DOI : 10.1021/acs.bioconjchem.8b00180.

Labit E., Rabiller L., Rampon C., Guissard C., André M., Barreau C., Cousin B., Carrière A., Eddine M.A., Pipy B., Pénicaud L., Lorsignol A., Vriz S.*, Dromard C.* et CASTEILLA L.*, "Opioids prevent regeneration in adult mammals through inhibition of ROS production », Scientific Reports, vol. 8, n 1, 2018, 12170, DOI : 10.1038/s41598-018-29594-1.

Roux L.N., Petit I., Domart R., CONCORDET J.-P., Qu J., Zhou H., Joliot A., FerRigno O. et ABERDAM D., « Modeling of aniridia-related keratopathy by CRISPR/Cas9 genome editing of human limbal epithelial cells and rescue by recombinant PAX6 protein », Stem Cells, vol. 36, no 9, 2018, p. 1421-1429, DOI : 10.1002/stem.2858. 